\title{
Mitteilungen der Deutschen Gesellschaft für
}

\section{Transfusionsmedizin und Immunhämatologie (DGTI)}

Gemeinsamer Kongreß der Deutschen Gesellschaft für Transfusionsmedizin und

Immunhämatologie (DGTI) und European Society for Haemapheresis (ESFH)

vom 8. bis 14. September 1991 in Würzburg

KONGRESS-ORGANISATION

European Society for Haemapheresis (ESFH)

Kongreßpräsident V. Kretschmer, Marburg, Transfusionsmedizin und Gerinnungsphysiologie, Klinikum der Philipps-Universität, Conradistraße, D-3550 Marburg, Tel. 06421/284490, Fax 06421/285655, 284487

Deutsche Gesellschaft für Transfusionsmedizin und Immunhämatologie (DGTI)

Kongreßpräsident D. Wiebecke, Würzburg, Abteilung für Transfusionsmedizin und Immunhämatologie, Universitätskliniken, Josef-Schneider-Str. 2, D-8700 Würzburg, Tel. 0931/2013269, Fax 0931/2012282

Internationales administratives Sekretariat (ESFH) PD. Dr. D. Weisshaar, BlutspendedienstHessen, Institut Kassel, Mönchebergstr. 48 E, Postfach 103909, 3500 Kassel, Tel. 0561/879336, Fax 0561/879339

ab 1. Juni 1991: Mönchebergstr. 57, Postfach 103909, D-3500 Kassel, Tel. 0561/8793-202, Fax $0561 / 875396$

Wissenschaftliches Sekretariat (ESFH und DGTI)

Prof. Dr. V. Kretschmer, Transfusionsmedizin und Gerinnungsphysiologie, Klinikum der Philipps-Universität, Conradistraße, D-3550 Marburg, Tel. 06421/284490, Fax 06421/285655, 284487 DGTI-Kongreß-Sekretariat

Prof. Dr. D. Wiebecke, Rosemarie Weitze, Monika Sietz, Abteilung für Transfusionsmedizin und Immunhämatologie, Universitätskliniken, Josef-Schneider-Str. 2, D-8700 Würzburg, Tel. 0931/2013269, Fax 0931/2012282 Ausstellungs-Organisation

KnaufKassel, Ausstellungen und Messebau GmbH, Im Grund 2-4, D-3501 Fuldatal 1, Tel. 0561/81050, Telex 56118175, Fax 0561/810530 Tagungsort

Bayerische Julius-Maximilians-Universität Würzburg, Philosophiegebäude, Am Hubland, D8700 Würzburg Einsendung von Abstracts

Abstracts müssen bis spätestens 31. Mai 1991 an das Wissenschaftliche Sekretariat, Prof. Dr. V. Kretschmer, unter Angabe der Fax-Nummer des Einsenders gerichtet werden. Das wissenschaftliche Komitee wird über die Annahme der Abstracts entscheiden und alle angenommenen in Infusionstherapie (Karger, Basel/München) publizieren.

\section{VORLÄUFIGES PROGRAMM}

Kongreßsprache

Deutscher Kongreß (DGTI): deutsch und englisch* Internationaler Kongreß (ESFH): englisch* Sonntag, 8. September 1991

A. Fortbildungsseminare für Medizinisch-technische Assistenten/innen 
Abbott-Workshop «Aktuelle Trends im Infektionsserologischen Labor»

Interne und externe Qualitätskontrolle in der Immunhämatologie

B. Fortbildungsseminare für Operatoren

Seminare der Firmen Haemonetics, Fresenius, Cobe und Baxter

Geschäftsstelle: Sandhofstraße 1 D-6000Frankfurt/M.71 Telefon 069/63015595

Wissenschaftliches Programm

Montag, 9. September 1991

- Filtration und Bestrahlung von Blutkomponenten

*Es erfolgt keine Übersetzung

151

I

Mitteílungen der

Transfusionsmedizinische Probleme bei Organtransplantation DeiltscheП Gesellschaft

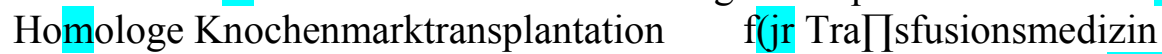

Biotechnik und immunhämatologische Diagnostik - Erythrozyten un(j Ir \munhämatoloeie

Therapie mit Zytokinen

Dienstag, 10. September 1991

Neue Methoden der Blutkomponentenpräparation und -lagerung

Biotechnik und immunhämatologische Diagnostik - Erythrozyten

Therapeutische Hämapheresen unter rheologischen Aspekten

Perioperative Gerinnungsdiagnostik

Plasmaproteine, Immunglobuline

Mittwoch, 11. September 1991

Infektionsdiagnostik

Biotechnik und immunhämatologische Methoden - Thrombozyten, Leukozyten

Autologe Hämotherapie

Neue Aspekte in der Histokompatibilitätsdiagnostik

Freie Vorträge

International Seminar on Haemapheresis

Internationale Seminare der Firmen Baxter, Cobe, Fresenius und Haemonetics

Donnerstag, 12. September 1991

Therapeutic haemapheresis in neurological dieseases

New apheresis techniques

Free communications

EDV-Automatisation

Immunhämatologie in der Schwangerschaft und Neonatalperiode

Freitag, 13. September 1991

Stem cells - Separation, purification, expansion and mobilisation

New aspects in platelet preparation, storage and transfusion Immune therapy, immune modulation

Der kritische Hämoglobin- und Hämatokritwert

Samstag, 14. September 1991

Virus inactivation of cellular blood products and plasma

Quality assurance in haemapheresis

Geschäftsstelle:

Sandhofstraße 1 
D-6000Frankfurt/M.71

Telefon 069/63015595 